\title{
Isoclinic Ambiguity Unwrapping of Circular Ring under Diametric Compression
}

\author{
M.-J. Huang ${ }^{1, a}$ \\ ${ }^{1}$ Mechanical Engineering, National Chung Hsing University, 40227 Taichung, Taiwan
}

\begin{abstract}
Isoclinic and isochromatic parameters in photoelastic analysis are formulated by arc tangent function of several phase shifting frames but for isochromatic calculation it is, in addition, in terms of isoclinic data also. Thus, any isoclinic phase ambiguity would also result in the phase inconsistencies of isochromatic calculation and cause unwrapping problems and difficulties. Many methods had been proposed to treat these kinds of isochromatic fringes but lots of treatments were needed for the correct retrieval. In this work, the isoclinic ambiguity problem is investigated and solved directly by a novel regional phase unwrapping technique. Once the isoclinic phase ambiguity problems are solved, a correct isoclinic result can be obtained. Then, substituting this result into the isochromatic formation yields an ambiguity free isochromatic phase map, which can be easily restored by any phase unwrapping algorithm. A stress frozen sample - circular ring under diametric compression verifies its effectiveness. Usually happened is that this kind of sample is with ambiguous isoclinic data which would cause phase ambiguities of the isochromatic formulation if not treated well first.
\end{abstract}

\section{Introduction}

There are two characteristics - principal stress direction and principal stress difference, which can be provided by photoelastic experiments with the so-called isoclinic and isochromatic fringes in correspondence. Both parameters are formulated by arc tan function of several phase shifting frames but for isochromatic calculation it is in terms of isoclinic data in addition. Thus, any isoclinic phase ambiguity would result in the phase inconsistencies of isochromatic calculation and cause problems and difficulties of its retrieval. Many methods [1-3] have been proposed to unwrap these kinds of isochromatic fringes with phase ambiguities. Lots of treatments are needed to circumvent the aforementioned ambiguity problems to do the retrieval. In this work, the isoclinic ambiguity problem is investigated and solved directly by a novel spatial phase unwrapping technique $[4,5]$. Provided the phase ambiguity problems are solved in the isoclinic data, its retrieval work will be very easy to be done and thus a correct isoclinic result can be obtained. Substituting this result into the isochromatic formulation leads to an ambiguity free isochromatic phase map, which can be restored by any phase unwrapping algorithm. The spatial isoclinic unwrapping algorithm is applied on a stress frozen sample - circular ring under diametric compression to verify its effectiveness. On this sample there exist isotropic and singular points on it. Usually happened is that this kind of sample is

\footnotetext{
a e-mail : mjhuang@dragon.nchu.edu.tw
} 
with ambiguous isoclinic data which would cause phase ambiguities of the isochromatic formulation if not treated well first.

\section{Isoclinic formulation}

Two well known phase shifting algorithms $[6,7]$ are considered for the calculation of the isoclinic parameter - the Wang-Patterson algorithm and the Pinit-Umezaki algorithm which are formulated by circular and plane polariscope respectively and with their isoclinic calculations listed as Eq. (1) and (2).

$$
\begin{gathered}
\varphi_{w}=\frac{1}{2} \tan ^{-1}\left(\frac{I_{a} \sin \Delta \sin 2 \varphi}{I_{a} \sin \Delta \cos 2 \varphi}\right), \\
\varphi_{w}=\frac{\pi}{8}-\frac{1}{4} \tan ^{-1}\left(\frac{I_{a} \sin ^{2} \frac{\Delta}{2} \sin 4 \varphi}{I_{a} \sin ^{2} \frac{\Delta}{2} \cos 4 \varphi}\right),
\end{gathered}
$$

where $\varphi_{w}$ and $\varphi$ are the wrapped and true isoclinic data respectively, $I_{a}$ is light intensity accounting for the amplitude of light, and $\Delta$ is the isochromatic data. It is clearly shown that there are common factors (i.e., $\sin \Delta$ and $\sin ^{2} \Delta / 2$ for Eq. (1) and (2), respectively) simultaneously existing in the numerator and denominator of the above equations and that could cause undefined region when their values approach zero. Pinit and Umezeki [7] suggested a multi-wavelength compensation method which will be adopted in the following isoclinic calculation to circumvent the undefined problems mentioned above.

Fig. 1 shows the schematic diagram of the plane polariscope used in this work for the phase stepping isoclinic calculation. Dark field setup is used and its four phase shifting frames are set as $\beta$ angle equals $0, \pi / 8, \pi / 4$, and $3 \pi / 8$, respectively and the four phase shifting light intensities are formulated as Eqs. (3a) (3d).

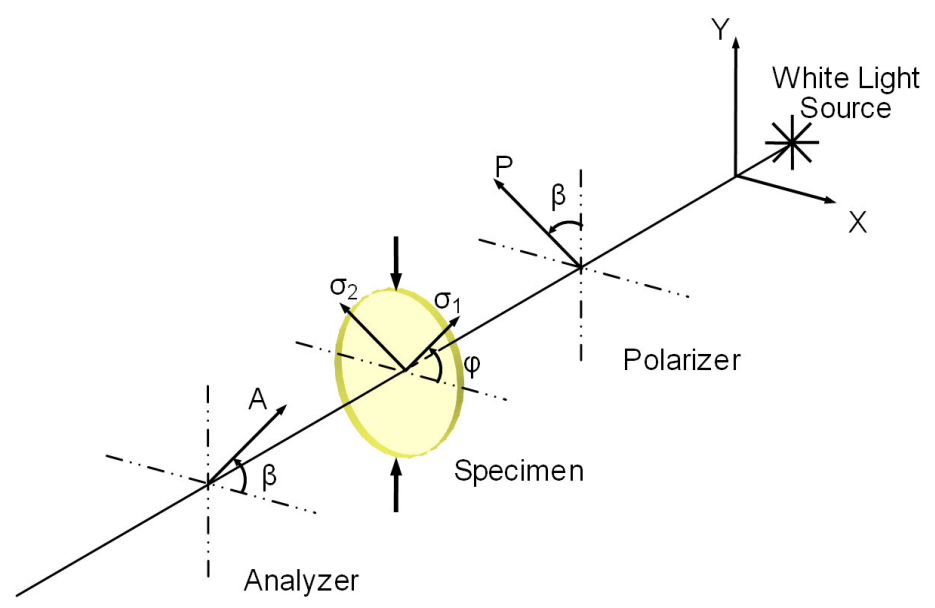

Fig. 1. The plane polariscope used for the isoclinic calculation. 


$$
\begin{aligned}
& I_{1, i}=I_{b, i}+\frac{1}{2} I_{a, i} \sin ^{2} \frac{\Delta}{2}(1-\cos 4 \varphi), \\
& I_{2, i}=I_{b, i}+\frac{1}{2} I_{a, i} \sin ^{2} \frac{\Delta}{2}(1-\sin 4 \varphi), \\
& I_{3, i}=I_{b, i}+\frac{1}{2} I_{a, i} \sin ^{2} \frac{\Delta}{2}(1+\cos 4 \varphi), \\
& I_{4, i}=I_{b, i}+\frac{1}{2} I_{a, i} \sin ^{2} \frac{\Delta}{2}(1+\sin 4 \varphi) .
\end{aligned}
$$

$\mathrm{I}_{1, \mathrm{i}} \sim \mathrm{I}_{4, \mathrm{i}}$ are the four phase stepping frames and the second sub index i stands for R (red), $\mathrm{G}$ (green) and $B$ (blue) images of the color digital camera under white light illumination. $I_{b}$ and $I_{a}$ are respectively the background light intensity and light amplitude of the plane polariscope. $\varphi$ and $\Delta$ are of the same physical meanings as they have appeared in Eqs. (1) and (2). The modified isoclinic formulation is as follows.

$$
\begin{aligned}
\varphi_{w} & =\frac{1}{4} \tan ^{-1}\left\{\frac{n<I_{4}^{s}-I_{0}>-n<I_{2}^{s}-I_{0}>}{n<I_{3}^{s}-I_{0}>-n<I_{1}^{s}-I_{0}>}\right\} \\
& =\frac{1}{4} \tan ^{-1}\left\{\frac{\left(\sin ^{2} \frac{\Delta_{R}}{2}+\sin ^{2} \frac{\Delta_{G}}{2}+\sin ^{2} \frac{\Delta_{B}}{2}\right) \sin 4 \varphi}{\left(\sin ^{2} \frac{\Delta_{R}}{2}+\sin ^{2} \frac{\Delta_{G}}{2}+\sin ^{2} \frac{\Delta_{B}}{2}\right) \cos 4 \varphi}\right\} \\
= & \frac{1}{4} \tan ^{-1}\left\{\frac{\sin 4 \varphi}{\cos 4 \varphi}\right\} .
\end{aligned}
$$

where

$$
I_{0}=\frac{1}{4}\left(I_{1}^{s}+I_{2}^{s}+I_{3}^{s}+I_{4}^{s}\right)
$$

and

$$
\begin{aligned}
& I_{j}^{s}=\frac{1}{3}\left(I_{j, R}+I_{j, G}+I_{j, B}\right), \quad \text { for } j=1 \sim 4, \\
& n<I>=\frac{I-\min (I)}{\max (I)-\min (I)} .
\end{aligned}
$$

$\mathrm{N}<>$ represents a normalization calculation and will convert all image data into an interval ranging between 0 and 1 . Since isoclinic calculation should be independent of light wavelength it used, thus if there exists any undefined term resulted from null sine square term in either one of the wavelengths of RGB, the summation operation of which in Eq. (4) will automatically average out the undefined result. In addition, the $\sin ^{2} \Delta / 2$ term multiplier won't change the signs of both of the numerator and denominator, therefore, the arctangent operation can be expanded into a $2 \pi$ full module and the calculated wrapped isoclinic is thus spanning over a $\pi / 2$ interval.

\section{Phase ambiguity and branch cuts}

Phase map obtained either by Eqs. (1) or (2) should be checked for its phase ambiguities (also called phase inconsistencies) before unwrapping algorithm is applied on. Phase inconsistency detection is applied to the isoclinic wrapped map to find whether there exists any phase inconsistency on the phase map or not. The phase inconsistency detection rule [8] is formulated as follows. 


$$
\begin{gathered}
\phi_{x}(m, n)=\phi(m, n+1)-\phi(m, n), \\
\phi_{y}(m, n)=\phi(m+1, n)-\phi(m, n), \\
N(m, n)=\operatorname{int}\left[\frac{\phi_{x}(m, n)}{2 \pi}\right]+\operatorname{int}\left[\frac{\phi_{y}(m, n+1)}{2 \pi}\right]-\operatorname{int}\left[\frac{\phi_{x}(m+1, n)}{2 \pi}\right]-\operatorname{int}\left[\frac{\phi_{y}(m, n)}{2 \pi}\right] .
\end{gathered}
$$

The "int" operator is an operator that rounds the value of the bracket to its nearest integer. A 2 × 2 inconsistency check is performed for every point $(\mathrm{m}, \mathrm{n})$ on the phase map. Provided the result of $\mathrm{N}$ $(\mathrm{m}, \mathrm{n})$ equals zero, the point $(\mathrm{m}, \mathrm{n})$ is an inconsistency-free point. A positive or negative inconsistency is existed depending on the result of $\mathrm{N}(\mathrm{m}, \mathrm{n})$ is +1 or -1 . If any phase inconsistency is detected, building branch cuts are further necessary to disable the incorrect integration path to avoid any inconsistent crossing in the phase retrieval work. Pairing algorithm can be referred to Reference works [9-12]. Provided the branch cut works are correctly done, regional phase unwrapping algorithm $[5,13]$ can be further used for the efficient work of phase unwrapping.

The unwrapping algorithm of isoclinic data is listed as follows.

(a) Use Eq. (4) to achieve the isoclinic wrapped map.

(b) Add $\pi / 2$ to all negative isoclinic data to convert calculated wrapped data of the previous step into a range of $0 \sim \pi / 2$.

(c) Find all the phase inconsistencies points of step (b).

(d) Build branch cuts on the phase inconsistencies to avoid any non-conservative phase integration.

(e) Detect all the $\pi / 2$ phase discontinuities boundaries (with branch cut repair) which divide the wrapped map into different regions.

(f) Designate two labeled numbers (e.g., 1 and 2) alternatively to the neighboring regions of the previous step. For example, set the biggest area with label number 1, then its neighboring areas with label number 2. Pick out the largest one from the neighboring regions with latest labeled number 2 and find its neighboring areas then set label number 1 to these unlabeled neighboring areas. Repeat the rule on and on till all the regions are labeled.

(g) Subtract $\pi / 2$ to all the isoclinic data on regions with label number 1 to yield the first associated unwrapped result. Similarly, subtract $\pi / 2$ to all the isoclinic data on regions with label number 2 to yield the other associated unwrapped result.

(h) Find the area of step (a) with isoclinic data ranging in the region of $-\pi / 8$ to $+\pi / 8$.

(i) Check each individual of step (g) respectively with the result of step (h) to determine the total number of pixel on which both data of $(\mathrm{g})$ and $(\mathrm{h})$ are correspondingly the same.

(j) The two results of step (g) are the two in-plane principal stress directional maps of the tested model with the one in higher matching number of pixel as the first principal stress direction.

\section{Experimental works}

A stress frozen sample of circular disk under diametric compression is conducted and its wrapped isoclinic data is shown in Fig. 2(a) with its corresponding $0 \sim \pi / 2$ distribution shown as Fig. 2(b). Phase inconsistencies are detected and residues pairing algorithm are applied on to build branch cuts and thus avoiding any integration across them. Regional partition is thus generated and its alternative region labeling is shown in Fig. 2 (c). Adding $\pi / 2$ to all the phase data of those light color regions of Fig. 2(c) yields the result of Fig. 2(d), which is the first principal direction distribution of the tested sample. 


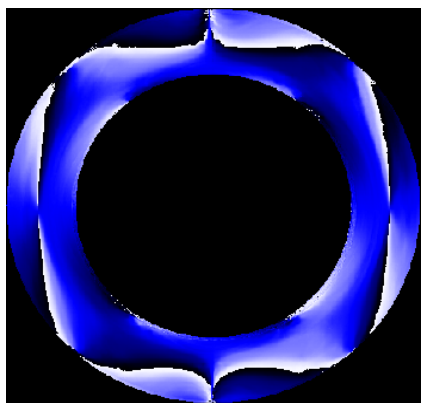

(a)

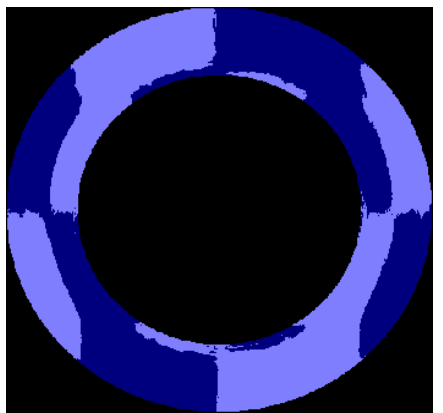

(c)

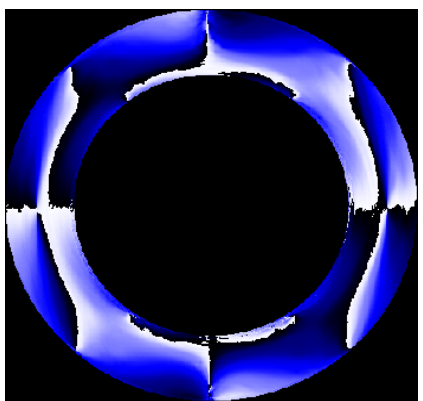

(b)

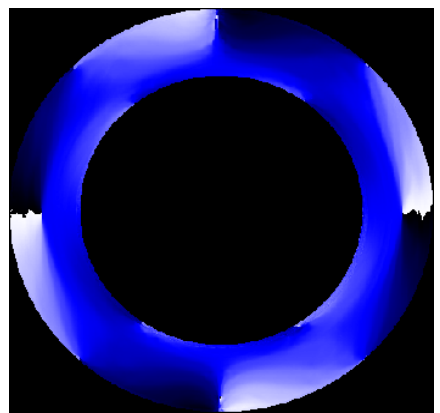

(d)

Fig. 2. The experimental study of a circular ring under diametric compression.

\section{Conclusions}

A simple and effective isoclinic phase inconsistencies removal technique is proposed and has been proven to be effective for the phase unwrapping of digital photoelasticity. Without having to find the isotropic points on the tested sample and the largest valid region as an initial work, the experimental results prove the effectiveness and robustness of the proposed algorithm. In addition, the proposed algorithm is very simple and can be implemented easily. Its regional retrieving characteristic also makes the unwrapping work very time effective.

Once the isoclinic data is correctly unwrapped, the isochromatic data can be obtained under substituting the unwrapped isoclinic data into the isochromatic formulation. Since the isoclinic data are correctly unwapped, the calculated isochromatic results are inconsistencies free. Therefore, most phase unwrpping algorithms can be further applied for its retrieval with difficulty. The proposed algorithm makes the unwrapping work of digital photoelasticity simple and straightforward. Only isoclinic data is treated first. Any ambiguity of the isoclinic map is blocked by the branch cut and that yields the correct isoclinic unwrapping work by the algorithm. Then, isochromatic formulation result is free of residue too and can be easily restored.

\section{References}

1. P. Pinit, E. Umezaki, Optics and Lasers in Engineering 45, 795 (2007)

2. V.S. Prasad, K.R. Madhu, K. Ramesh, Optics and Lasers in Engineering 42, 421 (2004)

3. K. Ashokan, K. Ramesh, Measurement Science and Technology, 17, 2891 (2006) 
4. M.J. Huang, B.C. Song, Proceeings of the 2009 SEM Annual Conference and Exposition of Experimental and Applied Mechanics, June 1-4, Albuquerque, New Mexico USA (2009)

5. M.J. Huang and Po-Chi Sung, Optics Express, 18, 1419 (2010)

6. Z.F. Wang and E.A. Patterson, Optics and Laser in Engineering, 22, 91 (1995)

7. P. Pinit and E. Umezaki, Optics and Laser in Engineering, 45, 795 (2007)

8. D.C. Ghiglia, G.A. Mastin, and L.A. Romero, JOSA, 4, 267 (1987)

9. R.M. Goldstein, H.A. Zebker, and C. Werner, Radio Sci., 23, 713 (1988)

10. T.J. Flynn, JOSA, 14, 2692 (1997)

11. D.C. Ghiglia and M.D. Pritt, Two-dimensional phase unwrapping theory, algorithms, and software (John Wiley \& Sons, Inc., New York, 1998)

12. Z. Wang and S. Li, Applied Optics, 38, 805 (1999)

13. J.J. Gierloff, SPIE, 818, 2 (1987). 